\title{
Self-Fulfilling Diagnoses
}

\section{George Baran*}

Temple University College of Engineering, 1947 N. 12th Street, Philadelphia, 19122, USA

${ }^{*}$ Corresponding author: George Baran, Temple University College of Engineering, 1947 N. 12th Street, Philadelphia, 19122, USA, Tel: (215)-204-8824; E-mail: GRBaran@Temple.edu

Received date: April 20, 2016; Accepted date: April 21, 2016; Published date: April 25, 2016

Citation: Baran G (2016) Self-Fulfilling Diagnoses. J Clin Exp Orthop 2: 1. doi: 10.4172/2471-8416.100015

Copyright: (c) 2016 Baran G. This is an open-access article distributed under the terms of the Creative Commons Attribution License, which permits unrestricted use, distribution, and reproduction in any medium, provided the original author and source are credited.

A young lady 23 years of age (let's call her D), recently reported to her orthopedic surgeon complaining of pain in the right hip. Because it was summertime and warm, she wore shorts to the appointment. An obvious rash could be seen in the area where there was pain. An X-ray revealed no contributing factors. The surgeon prescribed physical therapy, with the comment that if the pain did not resolve, an MRI would be ordered.

Four days later, the pain had intensified to the degree that the patient fainted at work and was sent to the ER. An extensive work-up including MRI and ultrasound took all day, yet there was no diagnosis made by the team of ER physicians. The patient was sent home with a prescription for analgesics, and advised to wait.

The next day, " $\mathrm{D}$ " walked into a local clinic because the discomfort associated with the rash persisted and she hoped that at least this condition could be treated. The primary care physician on call took one look and immediately diagnosed herpes zoster. The diagnosis explained the symptoms: pain, rash, and itch. Unfortunately, the diagnosis was made too late for an effective course of antivirals and " $D$ " continued to suffer for another few weeks although the pain and itch steadily diminished in severity.

In this case, a number of specialty physicians did not correctly diagnose the case, and appeared to ignore the presence of the rash at the site of pain. Though herpes zoster is unusual in young women, a thorough history would have revealed that as a child, " $D$ " had contracted an extremely mild case of varicella and was therefore at somewhat increased risk of developing herpes zoster.

Importantly perhaps, the physicians were not tuned to think outside the "box" of their own specialty. A recent report by the Institute of Medicine, "Improving Diagnosis in Health Care", makes the point that diagnostic errors are experienced by 5\% of adults seeking outpatient care each year. These errors are caused by a variety of contributing factors, including difficulty in accessing patient records, and poor or non-existent teamwork between health professionals. The Canadian Medical Protective Association (P-1205-3-E, 2012) has also identified cognitive bias, or the tendency for "anchoring (focusing on one symptom or diagnosis and failing to consider other possibilities), premature closure (uncritical acceptance of an initial diagnosis), and search satisfaction (calling off the search when just one abnormality has been found)". A report by Hashem, Chi and Friedman in J Biomedical Informatics 2003 confirmed the hypothesis that specialty physicians tended to "pull" cases into their specialty.

No doubt all physicians strive for absolute accuracy in diagnosis, and no doubt each one will recall an instant when they got it wrong. A physician's increased awareness of cognitive or specialty bias as a confounding factor in diagnoses will reduce the incidence of diagnostic error in the future. 